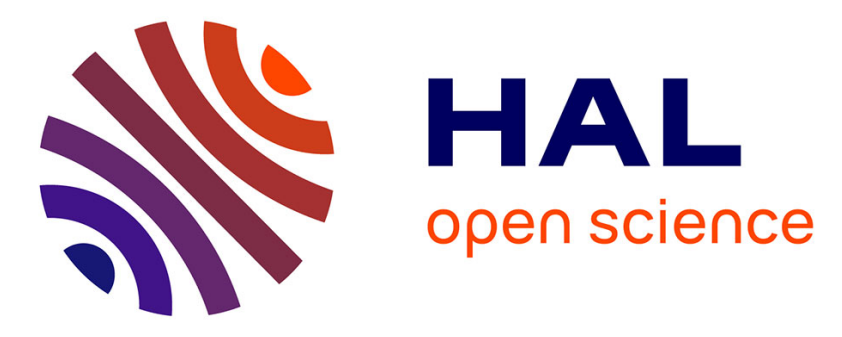

\title{
An event-triggered observer based control strategy for SISO systems
}

Daniel G. Sbarbaro, Sophie Tarbouriech, João Manoel Gomes da Silva Jr

\section{To cite this version:}

Daniel G. Sbarbaro, Sophie Tarbouriech, João Manoel Gomes da Silva Jr. An event-triggered observer based control strategy for SISO systems. IEEE 53rd Annual Conference on Decision and Control (CDC 2014), Dec 2014, Los Angeles, United States. pp.2789 - 2794, 10.1109/CDC.2014.7039817 . hal-01856297

\section{HAL Id: hal-01856297 https://hal.laas.fr/hal-01856297}

Submitted on 10 Aug 2018

HAL is a multi-disciplinary open access archive for the deposit and dissemination of scientific research documents, whether they are published or not. The documents may come from teaching and research institutions in France or abroad, or from public or private research centers.
L'archive ouverte pluridisciplinaire $\mathbf{H A L}$, est destinée au dépôt et à la diffusion de documents scientifiques de niveau recherche, publiés ou non, émanant des établissements d'enseignement et de recherche français ou étrangers, des laboratoires publics ou privés. 


\title{
An event-triggered observer based control strategy for SISO systems
}

\author{
D. Sbarbaro, S. Tarbouriech, and J. M. Gomes da Silva Jr.
}

\begin{abstract}
This work addresses the problem of devising a simple event-triggering strategy for observable and controllable SISO systems. The solution considers an observer based controller and an eventtriggering strategy taking into account solely local variables. Sufficient conditions to ensure the global stability of the event-triggering strategy are derived in terms of linear matrix inequalities (LMIs). Two simulation examples illustrate the application of the proposed method to both stable and unstable systems.
\end{abstract}

\section{INTRODUCTION}

Nowdays the implementation of modern control system requires the use of digital communication networks using both wired and wireless technologies. In this context aperiodic event- and self-triggering strategies have been proposed to deal with issues such as: communication, energy and computation constraints [1]. In particular, in many distributed applications the point of measurement is geographically separated from the location of the control processing. The sensor information is therefore sent by a wireless network, where the energy consumption can be a critical issue, since these devices are in general feed by batteries. Indeed, there are peaks of energy consumption for transmission/reception of data. Hence, reduce the sampling activity, i.e., the instants where the measurement information is transmitted is of great importance. On the other hand, in classical networks, it can be of interest to reduce the number of messages sent through the network, alleviating in this way the traffic and problems with delays and package losses.

Self-triggering strategies choose the sampling instant based on the available measurements and on predictions of the plant response. On the other hand, event-triggering controllers consider only the current measurements in order to define the sampling instant. Self-triggering strate-

This work was supported by ANR project LimICoS contract number 12 BS03 005 01, HYCON2 Network of Excellence grant agreement 257462, ADNEC project STIC-AmSud grant number 13STIC-03 from CAPES (Brazil), Conycit (Chile) and CNRS (France).

D. Sbarbaro is with the Department of Electrical Engineering. Universidad de Concepcion, Concepcion, Chile. dsbarbar@udec.cl

S. Tarbouriech is with CNRS, LAAS, 7 avenue du Colonel Roche, F-31400 Toulouse, France and Univ de Toulouse, LAAS, F-31400 Toulouse, France. tarbour@laas.fr

J. M. Gomes da Silva Jr. is with the UFRGS - Department of Electrical Engineering, Av. Osvaldo Aranha 103, 90035-190 Porto Alegre-RS, Brazil.jmgomes@ece.ufrgs.br. He is also supported by CNPq (Brazil) grants 30.6210/2009-6 (PQ) and 480638/2012-8 (Universal). gies for observer based controllers have been proposed in [2] based on a cascade interconnection of a discrete-time observer and a controller designed for state feedback. This approach has also been extended to deal with interconnected systems [3].

For event-triggered strategies, [4] proposes the use of a state observer in the event generator and an upper bound on the estimation error for designing an event triggering mechanism to guarantee a stable closed-loop system. A more general approach is proposed by [5], where three architectures for dynamic output feedback controllers are presented. The event-triggering conditions depend only on the norms of the local variables and are obtained using Lyapunov arguments. Dynamic output feedback controllers are also addressed in [6], where the asymptotic stability of the resulting closed-loop system is guaranteed by a condition in terms of an LMI (Linear Matrix Inequality) and in [7] these results are extended to deal with uncertain systems.

In this paper, we consider a Lyapunov approach, similarly to [8] and [9], to deal with the design of the eventtriggereing strategy for observer based state feedback controllers. In this context, as the plant evolves continuously, whereas the control signal is updated depending on discrete-time events, event-triggered control systems can be cast as hybrid or impulsive systems. Nevertheless, instead of considering the classical hybrid framework to study mixed continuous and discrete dynamics as defined in [10], we use an alternative direction as proposed in [11]. The approach we follow presupposes that a state feedback and an observer are designed, considering the separation principle. Then, an event-triggering strategy is proposed based on the decrease of a Lyapunov function. A key feature of the approach is that it only requires available measurable information. Moreover, the stability of the closed-loop sampled data system under the eventtriggering sampling strategy is formally proven based on the Lyapunov theory.

This paper is organized as follows: Section 2 states the problem to be addressed. Section 3 presents the observer based state feedback controller design in a continuoustime context. Section 4 is then dedicated to the eventtriggering strategy. Section 5 illustrates the implementation of this strategy to control both stable and unstable systems. Finally, Section 6 provides some final remarks and hints for future works.

Notation. For any matrix $A$. $A^{\prime}$ denotes the transpose. $H e\{A\}=A+A^{\prime}$. For two symmetric matrices of the 
same dimensions, $A$ and $B, A>B$ means that $A-B$ is positive definite. $I$ and 0 denote respectively the identity and the null matrix of appropriate dimensions.

\section{Problem Statement}

Consider the following continuous-time linear plant:

$$
\begin{aligned}
& \dot{x}_{p}(t)=A x_{p}(t)+B u(t) \\
& y_{p}(t)=C x_{p}(t)
\end{aligned}
$$

where $x_{p} \in \Re^{n}, u \in \Re, y_{p} \in \Re$ are the plant state, input and output, respectively. Matrices $A, B$ and $C$ are constant matrices of appropriate dimensions. In addition, the pair $(A, B)$ is controllable and the pair $(A, C)$ is observable.

Let us consider an observer based state feedback controller to drive the output to a given nonzero constant set-point $r$ :

$$
\begin{aligned}
\dot{x}_{o}(t) & =A x_{o}(t)+B u(t)+K_{o}\left(y(t)-y_{o}(t)\right) \\
y_{o}(t) & =C x_{o}(t), \\
u(t) & =K_{c} x_{o}(t)+K_{r} r .
\end{aligned}
$$

where $x_{o} \in \Re^{n}$ and $y_{o} \in \Re$ are the state and output of the observer, respectively. Furthermore, $K_{r} \in \Re$ is a feedforward gain, $K_{o} \in \Re^{n}$ and $K_{c} \in \Re^{n}$ are the observer and controller gains respectively. Design conditions regarding the three gains will be given in the next section.

In this paper, we are interested in looking at the eventtriggered implementation of the controller represented by (2). In particular, we consider a sensor node with the capability to estimate the state of the system and a controller based on the estimated state. The sensor and controller are in different nodes of the network as depicted in Figure 1. The block ST represents the eventtriggered sampling strategy.

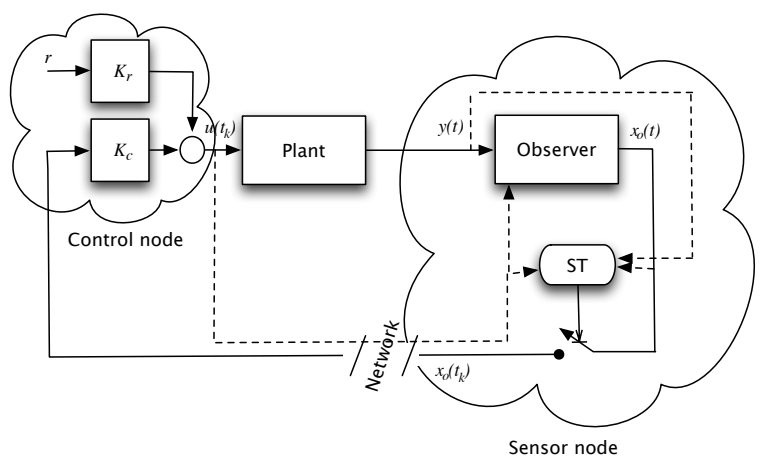

Fig. 1. Observer based controller.

The estimated state is sampled in discrete instants of time and the control action is supposed to be constant between two subsequent sampling instants $t_{k}$ and $t_{k+1}$. Note however, that differently from classical digital control approaches, the sampling interval $\left[t_{k}, t_{k+1}\right)$ is not constant. The system dynamics in the interval $\left[t_{k}, t_{k+1}\right)$ can therefore be described as follows:

$$
\begin{aligned}
& \dot{x}_{p}(t)=A x_{p}(t)+B u\left(t_{k}\right) \\
& \dot{x}_{o}(t)=A x_{o}(t)+B u\left(t_{k}\right)+K_{o} C \tilde{x}(t) \\
& u\left(t_{k}\right)=K_{c} x_{o}\left(t_{k}\right)+K_{r} r, \quad \forall t \in\left[t_{k}, t_{k+1}\right)
\end{aligned}
$$

where $\tilde{x}(t)=x_{p}(t)-x_{o}(t) \in \Re^{n}$ represents the state estimation error. The main aim of this work is to devise an event-triggered strategy to sample and to update the control signal applied to the plant based solely on available signals, that is, using only the signals $u(t), x_{o}(t)$ and $y_{p}(t)$.

With this aim, two steps should be followed. Firstly, the design of the observer and a state feedback control law should be carried out considering a continuous-time behavior of the closed-loop system and the achievement of some performance requirements. From this step a Lyapunov function, which certificates the asymptotic stability of the closed-loop system, is determined. The second step is the definition of the event-triggered strategy, which will be based on the Lyapunov function determined in the first step.

These steps are described in the following sections.

\section{Controller Design}

In this section we consider the continuous-time system described by (1)-(2). The control design is carried out according to the separation principle. The observer gain $K_{o}$ is designed to render $A+K_{o} C$ Hurwitz. Thus the equilibrium point $\tilde{x}_{e q}=0$ of the state estimation error dynamic:

$$
\dot{\tilde{x}}(t)=\left(A+K_{o} C\right) \tilde{x}(t)
$$

is globally asymptotically stable; i.e. $\lim _{t \rightarrow \infty} \tilde{x}(t)=0$, and the estimation output error

$$
\tilde{e}(t)=C \tilde{x}(t)
$$

also converges to zero; i.e. $\lim _{t \rightarrow \infty} \tilde{e}(t)=0$.

The dynamic of the observer state with the state feedback controller $u(t)=K_{c} x_{o}(t)+K_{r} r$ is given by:

$$
\dot{x}_{o}(t)=\left(A+B K_{c}\right) x_{o}(t)+B K_{r} r+K_{o} \tilde{e}(t)
$$

For a given constant reference signal $r$, the equilibrium point $\left(x_{p, e q}, x_{o, e q}\right)$ of (4) and (6) satisfies

$$
\begin{aligned}
& x_{o, e q}=x_{p, e q} \\
& \left(A+B K_{c}\right) x_{o, e q}+B K_{r} r=0 \\
& u_{e q}=K_{c} x_{o, e q}+K_{r} r
\end{aligned}
$$

This equilibrium point is then uniquely determined by $r$ provided that $\left(A+B K_{c}\right)$ is Hurwitz as follows:

$$
x_{o, e q}=-\left(A+B K_{c}\right)^{-1} B K_{r} r .
$$

The feedforward gain $K_{r}$ can be designed as $K_{r}=$ $-\left(C\left(A+B K_{c}\right)^{-1} B\right)^{-1}$ so that $y_{p, e q}=r$. The inverse of $C\left(A+B K_{c}\right)^{-1} B$ is well defined if there are no zeros at the origin for the closed-loop system. The dynamics of the 
error between the observer state $x_{o}(t)$ and its equilibrium point value, $\bar{x}_{o}(t)=x_{o}(t)-x_{o, e q}$, is given by

$$
\dot{\bar{x}}_{o}(t)=\left(A+B K_{c}\right) \bar{x}_{o}(t)+K_{o} \tilde{e}(t)
$$

where $\tilde{e}(t)$ can be interpreted as an input. The controller gain $K_{c}$ is designed so that $A+B K_{c}$ is Hurwitz and therefore one can render (9) input-to-state stable with respect to $\tilde{e}$. In other words, if $\tilde{e}$ is bounded then the state, $\bar{x}_{o}$, will also be bounded [12]. The following theorem characterizes the stability conditions and provides a Lyapunov function for the composite system, obtained from the equations (4), (5) and (9), in terms of a single LMI.

Theorem 1: Consider given matrices $K_{o}, K_{c}, Q_{c}=$ $Q_{c}^{\prime}>0, Q_{o}=Q_{o}^{\prime}>0$, if there exist matrices $P_{o}=$ $P_{o}^{\prime}>0, P_{c}=P_{c}^{\prime}>0$ such that $\Omega<0$, where

$$
\begin{aligned}
& \Omega=\left\{\left[\begin{array}{cc}
P_{c}\left(A+B K_{c}\right)+\frac{Q_{c}}{2} & P_{c} K_{o} C \\
0 & P_{o}\left(A+K_{o} C\right)+\frac{Q_{o}}{2}
\end{array}\right]\right\},
\end{aligned}
$$

then the equilibrium point of the system composed by (4), (5) and (9) is asymptotically stable.

Proof: Consider the following candidate Lyapunov function:

$$
V=\bar{x}_{o}^{\prime} P_{c} \bar{x}_{o}+\tilde{x}^{\prime} P_{o} \tilde{x}
$$

with $P_{c}=P_{c}^{\prime}>0$ and $P_{o}=P_{o}^{\prime}>0$. Then, the timederivative of (11) along the trajectories given by (4), (5) and $(9)$ is

$$
\begin{aligned}
\dot{V} & =\bar{x}_{o}^{\prime} H e\left\{P_{c}\left(A+B K_{c}\right)\right\} \bar{x}_{o}+\tilde{x}^{\prime} H e\left\{P_{o}\left(A+K_{o} C\right)\right\} \tilde{x} \\
& +\bar{x}_{o}^{\prime} P_{c} K_{o} C \tilde{x}+\tilde{x}^{\prime} C^{\prime} K_{o}^{\prime} P_{c} \bar{x}_{o}
\end{aligned}
$$

Note that $\Omega$ defined in (10) allows to write:

$$
\left[\begin{array}{c}
\bar{x}_{o} \\
\tilde{x}
\end{array}\right]^{\prime} \Omega\left[\begin{array}{c}
\bar{x}_{o} \\
\tilde{x}
\end{array}\right]=\dot{V}+\bar{x}_{o}^{\prime} Q_{c} \bar{x}_{o}+\tilde{x}^{\prime} Q_{o} \tilde{x} .
$$

Hence if $\Omega<0$ one gets

$$
\dot{V}<-\left(\bar{x}_{o}^{\prime} Q_{c} \bar{x}_{o}+\tilde{x}^{\prime} Q_{o} \tilde{x}\right)<0 .
$$

Thus $V$ is a Lyapunov function for the system described by (4), (5) and (9). Hence, one can conclude that the equilibrium point $\left(x_{p, e q}, x_{o, e q}\right)$ of the system composed by (4), (5) and (9) is asymptotically stable.

It is important to note that the Lyapunov function $V$ given in (11), cannot be directly used to design eventtriggering strategies, since the state estimation error is not available. In order to find useful conditions, that can be directly used in the design of implementable eventtriggering strategies, we explore the fact that the closedloop system, described by equations (4), (5) and (9), has a cascade structure. Thus, the following theorem provides a simplified stability condition along with a Lyapunov function.

Theorem 2: Consider the system composed by (4), (5) and (9) with fixed matrices $K_{o}$ and $K_{c}$, and matrices $Q_{o}=Q_{o}^{\prime}>0, Q_{c}=Q_{c}^{\prime}>0, Q_{e}=Q_{e}^{\prime}>0$, if there exist
$P_{c}=P_{c}^{\prime}>0$ and $P_{o}=P_{o}^{\prime}>0$ satisfying the following conditions

$$
\begin{gathered}
H e\left\{P_{o}\left(A+K_{o} C\right)\right\}+Q_{o e}<0 \\
{\left[\begin{array}{cc}
H e\left\{P_{c}\left(A+B K_{c}\right)\right\}+Q_{c} & P_{c} K_{o} \\
K_{o}^{\prime} P_{c} & -Q_{e}
\end{array}\right]<0}
\end{gathered}
$$

with $Q_{o e}=Q_{o}+C^{\prime} Q_{e} C$, then the equilibrium point; i.e. the origin, of the composite system is asymptotically stable.

Proof: Let $V_{1}=\tilde{x}^{\prime} P_{o} \tilde{x}$ a Lyapunov candidate function for system (4). Its time-derivative along the trajectories of (4) is given by:

$$
\dot{V}_{1}=\tilde{x}^{\prime} H e\left\{P_{o}\left(A+K_{o} C\right)\right\} \tilde{x} .
$$

By using condition (15) one gets

$$
\dot{V}_{1}+\tilde{x}^{\prime} Q_{o e} \tilde{x}<0 \text {. }
$$

Thus $V_{1}$ is a Lyapunov function for system (4).

Let $V_{2}=\bar{x}_{o}^{\prime} P_{c} \bar{x}_{o}$ a quadratic function associated to system (9). Its time-derivative along the trajectories of (9) is given by:

$$
\begin{aligned}
\dot{V}_{2}= & \bar{x}_{o}^{\prime} H e\left\{P_{c}\left(A+B K_{c}\right)\right\} \bar{x}_{o}+\bar{x}_{o}^{\prime} P_{c} K_{o} \tilde{e}+ \\
& \tilde{e}^{\prime} K_{o}^{\prime} P_{c} \bar{x}_{o} .
\end{aligned}
$$

By using condition (16) it follows that

$$
\dot{V}_{2}+\bar{x}_{o}^{\prime} Q_{c} \bar{x}_{o}-\tilde{e}^{\prime} Q_{e} \tilde{e}<0 .
$$

Let us now consider the composite quadratic function $V=V_{1}+V_{2}$. Using (18) and (20), $\dot{V}$ can be bounded as follows

$$
\dot{V}=\dot{V}_{1}+\dot{V}_{2}<\tilde{e}^{\prime} Q_{e} \tilde{e}-\tilde{x}^{\prime} Q_{o e} \tilde{x}-\bar{x}_{o}^{\prime} Q_{c} \bar{x}_{o} .
$$

Thus, by replacing $Q_{o e}=Q_{o}+C^{\prime} Q_{e} C$, one obtains

$$
\dot{V}<-\left(\bar{x}_{o}^{\prime} Q_{c} \bar{x}_{o}+\tilde{x}^{\prime} Q_{o} \tilde{x}\right)<0 .
$$

Hence the quadratic function $V=V_{1}+V_{2}$ is a Lyapunov function of the composite system given by (4), (5) and (9). Then, one can conclude that the equilibrium point; i.e. the origin, of the composite system (4), (5) and (9) is asymptotically stable.

\section{Event-TRIGGERED STRATEGy}

In this section we propose an event-triggered implementation of the observer based state feedback controller issued from Theorem 2 .

Considering variables $\bar{x}_{o}, u$ and $\tilde{e}$, the observer equation in (9), for $t \in\left[t_{k}, t_{k+1}\right)$, can be rewritten as follows:

$$
\dot{\bar{x}}_{o}(t)=A \bar{x}_{o}(t)+B \bar{u}_{s}(t)+K_{o} \tilde{e}(t)
$$

with $\bar{u}_{s}(t)=u\left(t_{k}\right)-u_{e q}$, where from (7) recall that $u_{e q}=$ $K_{c} x_{o, e q}+K_{r} r$.

Define now the vector

$$
\xi(t)=\left[\begin{array}{lll}
\bar{x}_{o}(t)^{\prime} & \bar{u}_{s}(t) \quad \tilde{e}(t)
\end{array}\right]^{\prime} \in \Re^{n+2}
$$


and the matrix

$$
\mathcal{M}=\left[\begin{array}{ccc}
P_{c} A+A^{\prime} P_{c}+Q_{c} & P_{c} B & P_{c} K_{o} \\
B^{\prime} P_{c} & 0 & 0 \\
K_{o}^{\prime} P_{c} & 0 & -Q_{e}
\end{array}\right] .
$$

The event-triggered sampling strategy can therefore be described by using the following algorithm to define the sampling event:

Algorithm 1:

if $\xi^{\prime}(t) \mathcal{M} \xi(t)>0$ then sample, i.e:

$$
\begin{aligned}
& t_{k+1}=t \\
& k \leftarrow k+1 \\
& \bar{u}_{s}(t)=u(t)-u_{e q}
\end{aligned}
$$

otherwise:

$$
\bar{u}_{s}(t)=u\left(t_{k}\right)-u_{e q}
$$

The following theorem relies upon Theorem 2 to ensure the asymptotic stability of the closed-loop sampled-data system considering the event-strategy proposed in Algorithm 1.

Theorem 3: Consider the system composed by (4), (5) and (23), matrices $P_{o}$ and $P_{c}$ satisfying conditions of Theorem 2 and the event-triggered sampling strategy given in Algorithm 1, where $\mathcal{M}$ is defined as in (25). Then it follows $\lim _{t \rightarrow \infty} \bar{x}_{o}(t)=0$ and $\lim _{t \rightarrow \infty} \tilde{x}(t)=0$, or equivalently, the closed-loop system is asymptotically stable.

Proof: Let $V\left(\bar{x}_{o}, \tilde{x}\right)=V_{1}(\tilde{x})+V_{2}\left(\bar{x}_{o}\right)$ be a candidate Lyapunov function, where $V_{2}\left(\bar{x}_{o}\right)=\bar{x}_{o}^{\prime} P_{c} \bar{x}_{o}, V_{1}(\tilde{x})=$ $\tilde{x}^{\prime} P_{o} \tilde{x}$, and matrices $P_{o}$ and $P_{c}$ satisfying (15) and (16) respectively. Consider the following notation:

- $\dot{V}_{s}=\dot{V}_{1}(\tilde{x})+\dot{V}_{2 s}\left(\bar{x}_{o}, \bar{u}_{s}, \tilde{e}\right)$ for denoting the timederivative of $V$ along the trajectories of system (4), (5), (23)

- $\dot{V}=\dot{V}_{1}(\tilde{x})+\dot{V}_{2}\left(\bar{x}_{o}, \tilde{e}\right)$ for denoting the timederivative of $V$ along the trajectories of system (4), (5), (9).

By using (15) we have

$$
\dot{V}_{1}(\tilde{x})<-\tilde{x}^{\prime} Q_{o e} \tilde{x}, \quad \forall t .
$$

where $Q_{o e}=Q_{o}+C^{\prime} Q_{e} C>0$. From the definition of matrix $\mathcal{M}$ and vector $\xi$, it follows:

$$
\xi(t)^{\prime} \mathcal{M} \xi(t)=\dot{V}_{2 s}\left(\bar{x}_{o}, \bar{u}_{s}, \tilde{e}\right)+\phi\left(\bar{x}_{o}, \tilde{e}\right)
$$

where $\phi\left(\bar{x}_{o}, \tilde{e}\right)=\bar{x}_{o}^{\prime} Q_{c} \bar{x}_{o}-\tilde{e}^{\prime} Q_{e} \tilde{e}$. If $\xi(t)^{\prime} \mathcal{M} \xi(t) \leqslant 0, \forall t \in$ $\left[t_{k-1}, t_{k}\right)$, then one gets

$$
\dot{V}_{2 s}\left(\bar{x}_{o}, \bar{u}_{s}, \tilde{e}\right) \leqslant-\phi\left(\bar{x}_{o}, \tilde{e}\right), \quad \forall t \in\left[t_{k-1}, t_{k}\right)
$$

and therefore from (26) and (28)

$$
\begin{array}{r}
\dot{V}_{s}<-\bar{x}_{o}^{\prime} Q_{c} \bar{x}_{o}-\tilde{x}^{\prime} Q_{o} \tilde{x}<0 \\
\forall t \in\left[t_{k-1}, t_{k}\right) .
\end{array}
$$

Let us consider now the sampling instant $t=t_{k}$. At this instant, the control and the sensor values are instantaneously updated and it follows that:

$$
\bar{u}_{s}\left(t_{k}\right)=u\left(t_{k}\right)-u_{e q}=K_{c} \bar{x}_{o}\left(t_{k}\right)
$$

$A \bar{x}_{o}\left(t_{k}\right)+B \bar{u}_{s}\left(t_{k}\right)+K_{o} \tilde{e}\left(t_{k}\right)=\left(A+B K_{c}\right) \bar{x}_{o}\left(t_{k}\right)+K_{o} \tilde{e}\left(t_{k}\right)$

and

$$
\dot{V}_{2 s}\left(\bar{x}_{o}\left(t_{k}\right), \bar{u}_{s}\left(t_{k}\right), \tilde{e}\left(t_{k}\right)\right)=\dot{V}_{2}\left(\bar{x}_{o}\left(t_{k}\right), \tilde{e}\left(t_{k}\right)\right) .
$$

Suppose now that (16) is verified and from the proof of Theorem 2, it follows that:

$$
\dot{V}_{2 s}\left(\bar{x}_{o}\left(t_{k}\right), \bar{u}_{s}\left(t_{k}\right), \tilde{e}\left(t_{k}\right)\right)+\phi\left(\bar{x}_{o}\left(t_{k}\right), \tilde{e}\left(t_{k}\right)\right)<0 .
$$

Thus, we conclude that

$$
\xi\left(t_{k}\right)^{\prime} \mathcal{M} \xi\left(t_{k}\right)<0
$$

which means that the interval between two sampling instants is not empty. From this fact and from (4) and (9), it follows that the state converges asymptotically to the origin under the sampling strategy.

Note that the considered Lyapunov function is continuous at the sampling instants, so that the indicated properties are enough to conclude on asymptotic stability.

\section{Numerical examples}

Two simple simulation examples illustrate the strategy proposed in this work.

Example 1: The first example considers an open-loop stable system described by the following state space matrices:

$$
A=\left[\begin{array}{cc}
0 & 1 \\
-2 & -4
\end{array}\right], B=\left[\begin{array}{l}
0 \\
1
\end{array}\right], C=\left[\begin{array}{ll}
1 & 0
\end{array}\right] .
$$

The observer and controller parameters are defined as follows:

$$
K_{o}=-\left[\begin{array}{ll}
1 & 1
\end{array}\right]^{\prime}, K_{c}=-\left[\begin{array}{ll}
1 & 2
\end{array}\right], K_{r}=3 .
$$

Given matrices $Q_{c}$ and $Q_{e}$

$$
Q_{c}=\left[\begin{array}{ll}
1 & 0 \\
0 & 1
\end{array}\right], Q_{e}=10,
$$

the following values of matrices $P_{o}$ and $P_{c}$ are obtained by solving the LMIs (16) and (15):

$$
\begin{aligned}
P_{o} & =\left[\begin{array}{cc}
3.2778 & -1.5 \\
-1.5 & 0.8333
\end{array}\right] \\
P_{c} & =\left[\begin{array}{cc}
1.3832 & -0.6386 \\
-0.6386 & 0.4049
\end{array}\right]
\end{aligned}
$$

The simulation considers different initial conditions for the observer and the system and at time $t=10[\mathrm{~s}]$ an unit step change in the reference. The behavior of the controller is summarized in figure 2; where the sampling events are represented by a boolean variable $s_{e}$; i.e. $s_{e}=1$ if a sampling event occurs. As seen in figure 2 the strategy samples only when is necessary; i.e. once the system reaches the steady state no sampling is required. Figure 3 shows the evolution of the Lyapunov function and the switching condition. Notice that at every sampling event, the $\xi(t)^{\prime} \mathcal{M} \xi(t)$ is forced to be negative.

Example 2: The second example considers an unstable system having a pole in the right hand side of the complex plane. This type of structure arises for instance 

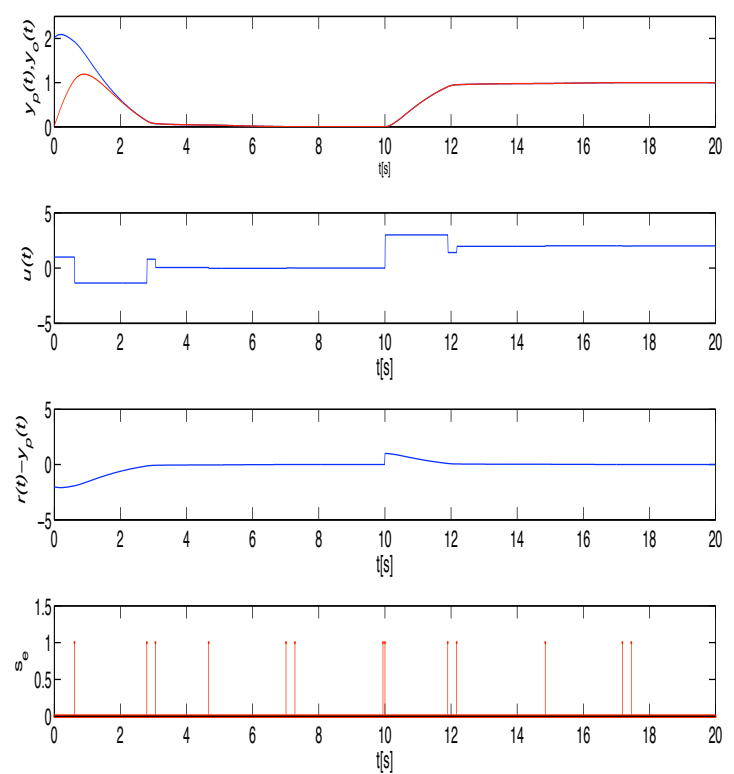

Fig. 2. Output of the continuous plant $y_{p}(t)$ (blue) and the estimated one $y_{o}(t)$ (red), control signal $u(t)$, control error $r(t)-y_{p}(t)$ and sampling events.

in modeling levitation systems. The parameters of the state space model are:

$$
A=\left[\begin{array}{ll}
0 & 1 \\
4 & 0
\end{array}\right], B=\left[\begin{array}{l}
1 \\
0
\end{array}\right], C=\left[\begin{array}{ll}
1 & 0
\end{array}\right] .
$$

The observer and controller parameters are defined by the following values

$$
K_{o}=-\left[\begin{array}{ll}
3.5 & 7
\end{array}\right]^{\prime}, K_{c}=-\left[\begin{array}{ll}
6 & 3
\end{array}\right], K_{r}=2 .
$$

Given matrices

$$
Q_{c}=\left[\begin{array}{ll}
1 & 0 \\
0 & 1
\end{array}\right], Q_{e}=1000
$$

and solving the LMIs (16) and (15) the following values for $P_{o}$ and $P_{c}$ are obtained:

$$
\begin{aligned}
P_{o} & =\left[\begin{array}{cc}
1506 & -1141 \\
-1141 & 1869
\end{array}\right] \\
P_{c} & =\left[\begin{array}{cc}
30.87 & 9.27 \\
9.27 & 33.41
\end{array}\right]
\end{aligned}
$$

As in the previous example, the simulation starts with a reference equal to zero and different initial conditions for the system and the observer, at time $t=10[s]$ an unit step change in the reference is simulated. As seen in figure 4, compared to the stable case of the previous example, the controller is able to stabilize the system by generating more sampling events, since in between sampling events the system is in fact in open-loop. Figure 5 shows the evolution of the Lyapunov function and the switching condition.
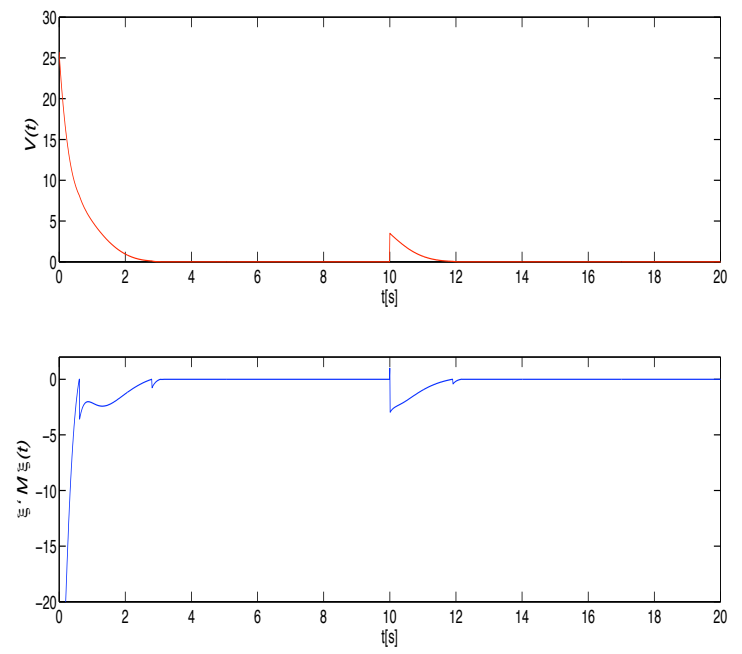

Fig. 3. Lyapunov function $V(t)$ and switching condition.

\section{Final Remarks}

In this paper a systematic method for designing eventtriggering strategies for observer-based controllers has been presented. The design of the observer and the controller parameters follows the separation principle. The event-triggering design method is based on Lyapunov and ISS arguments providing simple triggering strategies that only use information of available signals. Hence, since a Lyapunov-based approach is considered, the stability under the event-triggered sampling strategy is formally guaranteed. The proposed strategy has been illustrated by considering both stable an unstable systems, showing that the stability of the closed-loop system is ensured. Since the strategy is formulated using state space models, its extension to multi-input multi-ouput systems is straightforward for zero reference signals. For non zero references, however, the system must be square and the closed-loop system must not have transmission zeros at the origin in order to compute the feedforward gain $K_{r}$. The resulting event strategy will sample all the input and output signals at once.

In addition, the current work lets several directions of research open. Among them, it should be interesting to use additional constraints in order to enrich the stability conditions to decrease their conservatism, as, for example, some constraint about the sampling error. Furthermore, it should be challenging to consider the magnitude limitation of the actuator or of the sensor explicitly in the event-triggering method with the objective to characterize the domain of attraction around the equilibrium point (see [13] and [14] for some interesting directions on this). 

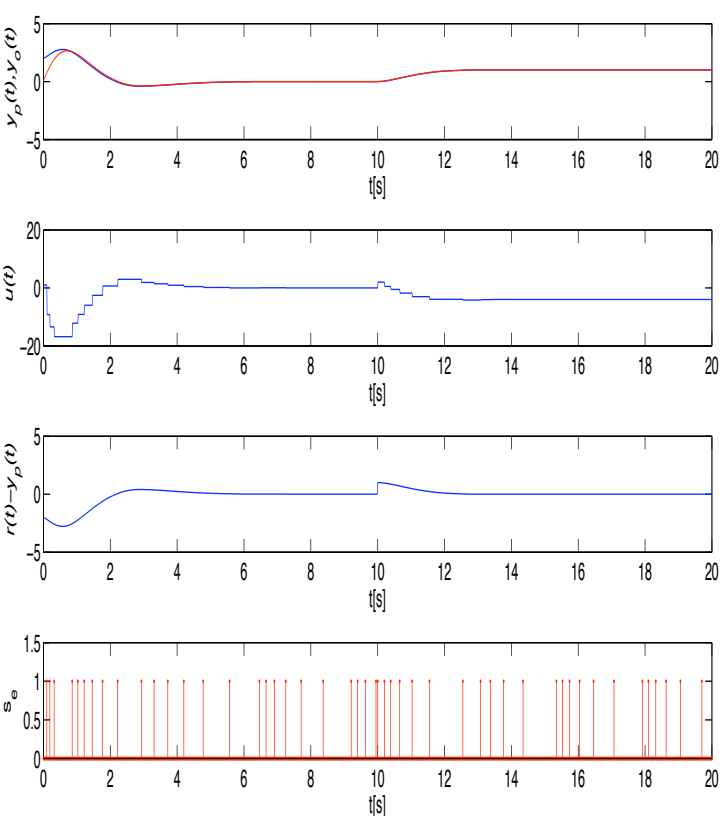

Fig. 4. Output of the continuous plant $y_{p}(t)$ (blue) and the estimated one $y_{o}(t)$ (red), control signal $u(t)$, control error $r(t)-y_{p}(t)$ and sampling events.

\section{REFERENCES}

[1] W.P.M.H. Heemels, K.H. Johansson, and P. Tabuada, "An introduction to event-triggered and self-triggered control," in Proceedings of the 51th IEEE Conference on Decision and Control, Maui, Hawaii, December 2012, pp. 3270 - 3285.

[2] J. Almeida, C. Silvestre, and A. M. Pascoal, "Observer based self-triggered control of linear plants with unknown disturbances," in American Control Conference, Montreal, Canada, June 2012, pp. 5688-5693.

[3] — - "Observer based self-triggered control of an acyclic interconection linear plants," in Proceedings of the 51th IEEE Conference on Decision and Control, Maui, Hawaii, December 2012, pp. $7553-7558$.

[4] D. Lehmann and J. Lunze, "Event-based output feedback control," in Proceedings of the 19th IEEE Mediterranean Conference on Control and Automation, Corfu, Greece, July 2011, pp. 982-987.

[5] P. Tallapragada and N. Chopra, "Event-triggered dynamic output feedback control of LTI systems," in Proceedings of the 51th IEEE Conference on Decision and Control, Maui, Hawaii, December 2012, pp. 6597-6602.

[6] X. Chen and F. Hao, "Stability of event-triggered outputfeedback control systems," in Proceedings of the 30th Chinese Control Conference, Yantai, Shandong, 2011, pp. 1184-1189.

[7] _ , "Observer based event-triggered control for certain and uncertain linear systems," IMA Journal of Mathematics Control and Information, pp. 1-16, 2013.

[8] A. Seuret and C. Prieur, "Event-triggered sampling algorithms based on a Lyapunov function," in 50th IEEE Conference on Decision and Control and European Control Conference (CDC-ECC), Orlando, Florida, 2011, pp. 6128-6133.

[9] N. Marchand, J.J. Martinez, S. Durand, and J. F. GuerreroCastellanos, "Lyapunov event-triggered control: a new event strategy based on the control," in Proceedings of the 9th IFAC
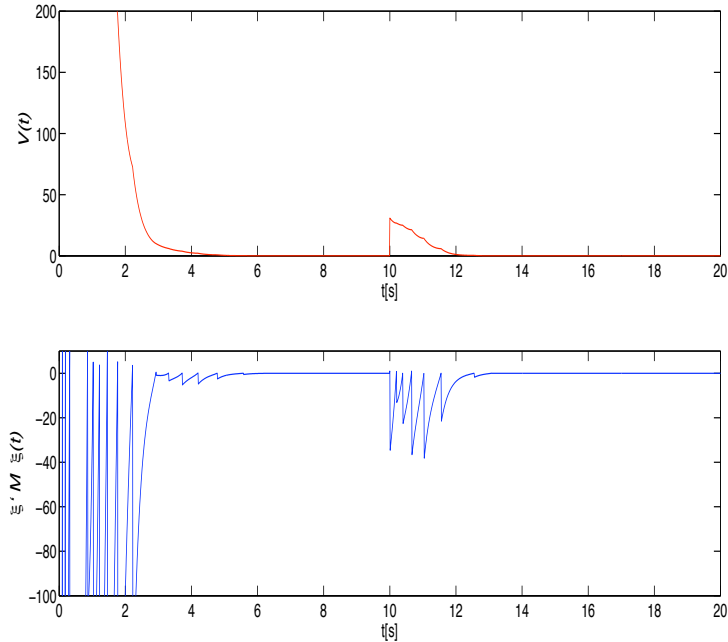

Fig. 5. Lyapunov function $V(t)$ and switching condition.

Symposium on Nonlinear Control Systems, Toulouse, France, 2013.

[10] R. Goebel, R. Sanfelice, and A. Teel, Hybrid Dynamical Systems. Modeling, Stability and Robustness. Princeton University, Press, 2012

[11] J.M. Gomes da Silva Jr., W. F Lages, and D. Sbarbaro, "Eventtriggered PI control design," in Proceedings of the 19th IFAC World Congress, Cape Town, South Africa, August 2014

[12] E.D. Sontag, Mathematical Control Theory - Deterministic finite dimensional systems. New York, USA: Springer-Verlag, 1990.

[13] A. Seuret, C. Prieur, S. Tarbouriech, and L. Zaccarian, "Eventtriggered sampling for linear closed-loop systems with plant input saturation," in Proceedings of the 9th IFAC Symposium on Nonlinear Control Systems (NOLCOS), Toulouse, France, Aug. 2013, pp. 341-346.

[14] G.A. Kiener, D. Lehmann, and K.H. Johansson, "Actuator saturation and anti-windup compensation in event-triggered control," Discrete Event Dynamic Systemss: Theory and Applications, vol. 24, no. 2, pp. 173-197, June 2014. 